UVX 2008 (2009) 15-19

(C) EDP Sciences, 2009

DOI: $10.1051 / \mathrm{uvx} / 2009004$

\title{
Shaping and characterization of tunable UV ultrashort pulses
}

\author{
S. Weber ${ }^{1}$, J. Bonnet $^{1}$, A. Besse ${ }^{1,2}$, A. Arbouet ${ }^{2}$ and B. Chatel ${ }^{1}$ \\ ${ }^{1}$ Laboratoire Collisions, Agrégats, Réactivité, IRSAMC, CNRS/Université Paul Sabatier \\ Toulouse 3, 31062 Toulouse Cedex 9, France \\ ${ }^{2}$ CEMES, CNRS/Université Toulouse, 29 rue Jeanne Marvig, BP. 94347, 31055 Toulouse, \\ France \\ e-mail: Beatrice@irsanc.ups-t/se.fr
}

\begin{abstract}
Time shaping of ultrashort UV pulses has been performed using a specially designed acoustooptic programmable dispersive filter in KDP material. A time window shaping up to 4 ps has been obtained on the $250-410 \mathrm{~nm}$ range. Characterization by difference frequency mixing as well as two-photon absorption in diamond has been used.
\end{abstract}

\section{INTRODUCTION}

The development of femtosecond laser technology has opened access to unforeseen applications in molecular, chemical physics, as well as biology [1-4]. In the past ten years, the active control of ultrafast physical or chemical processes by means of well-defined shaped laser pulses has become possible [5-7]. The high number of potential applications of femtosecond pulse shaping turned it very quickly into a very intense field of research.

Traditional methods for femtosecond pulse shaping are based on a Liquid Crystal Device (LCD) or Acousto-Optic Modulator (AOM) placed in the Fourier plane of a grating based zero dispersion 4f configuration [8-10]. The different wavelengths are spatially separated and can then be addressed individually. Spectacular results have been obtained with such devices [11,12]. The application of conventional programmable pulse shapers is restricted to the visible and near infrared spectral range because of the limited transparency range of common modulator materials, such as liquid crystals or $\mathrm{TeO}_{2}$. However quite recently interesting results have been obtained in the UV range by shaping in the IR and transfering the phase by sum frequency mixing or by directly shaping in the UV using deformable mirrors [13] or silica acousto-optic [14], placed in the Fourier plane of a $4 \mathrm{f}$ line. However changing the wavelength requires careful realignment, thus precluding easy tunability. On the other hand, a much simpler scheme, avoiding the complexity of the $4 \mathrm{f}$ line, and with higher efficiency, can be achieved using an Acousto-Optic Programmable Dispersive Filter (AOPDF) [15].

AOPDF are based on the propagation of light in an acousto-optic birefringent crystal. The interaction of an incident ordinary optical wave with a collinear acoustic shear wave leads to diffraction of an extraordinary wave. Spectral phase and amplitude pulse shaping of a femtosecond optical pulse can be achieved by controlling the amount of extraordinary versus ordinary propagation in the optical path of each of its spectral components. The collinear acousto-optic interaction and the reduced size result in an easy-to-align device, appropriate for insertion in an amplified laser chain or in a pump-probe setup. AOPDF have proven to be very useful to correct the time aberrations introduced in Chirped Pulse Amplifiers, for amplitude and phase control of ultrashort pulses $[15,16]$, or even in characterization set-ups [17].

Until recently, using $\mathrm{TeO}_{2}$ acousto-optic device, most of AOPDF applications were restricted to the near infrared. Recently they have been extanded to the visible range [18]. Indeed, the $\mathrm{TeO}_{2}$ transparency window does not allow to use this type of device for shorter wavelength. In 2006, Coudreau et al [19] have designed an AOPDF using KDP material for operation in the UV range. In their paper, a very nice proof of principle is done showing shaped spectrum. 
Here, we report on pulse compression and shaping between $255 \mathrm{~nm}$ and $400 \mathrm{~nm}$ using a newly designed AOPDF. This device provided us with around $60 \mathrm{fs}$ pulses on a 4 ps shaping window, easily tunable in the 253-408 $\mathrm{nm}$ range. The duration is only limited by the duration of the input pulses. Our laser system is a conventional amplified kilohertz Ti:Sa laser delivering $50 \mathrm{fs}$ pulses at $805 \mathrm{~nm}$. Three different sources of ultraviolet femtosecond pulses have been used in our experiments: the second and third harmonics of the infrared femtosecond pulses respectively at $408 \mathrm{~nm}$ (FWHM $6 \mathrm{~nm}$ ) and $268 \mathrm{~nm}$ (FWHM $2.7 \mathrm{~nm}$ ) and the output of a frequency-doubled Non-Collinear Optical Amplifier (NOPA) yielding UV pulses tunable between $250 \mathrm{~nm}$ and $350 \mathrm{~nm}$ (FWHM few $\mathrm{nm}$ depending on the central wavelength). In a first set of experiments, we demonstrated the ability of the AOPDF to yield precisely tailored pulses at $268 \mathrm{~nm}$ using cross-correlation characterization by DFG (difference frequency generation) in a BBO crystal. A second set addressed pulse shaping and compression of the second harmonic at $408 \mathrm{~nm}$ as well as the tunable output of the frequency-doubled NOPA. The novelty is to characterize these pulses by two-photon absorption in diamond, which doesn't require any phase matching condition. Different tailored pulses have been obtained and pulse compression down to $60 \mathrm{fs}$ at $408 \mathrm{~nm}$ has been unambiguously demonstrated.

\section{SHAPING AT $268 \mathrm{~nm}$ AND CHARACTERIZATION BY DIFFERENCE FREQUENCY MIXING}

Figure 1 shows several cross-correlation performed by DFG in a $150 \mu$ m-thick BBO crystal between the $268 \mathrm{~nm}$ going through the AOPDF and the IR reference. Multiple output pulses have been generated simply by summing multiple acoustic pulses. An example of a 7-pulse sequence is shown fig. 1(a) at $268 \mathrm{~nm}$. Some irregularities in the amplitude are visible. They could originate from complex acoustic or non linear optics effects in the KDP crystal. They are currently under careful investigation. However, one can compensate them by adjusting carefully the amplitude coefficient of each pulse. Quadratic phase as well as cubic phase have been also programmed successfully. The fig. 1(b) shows a sequence of two pulses one is Fourier limited, the second one is chirped.
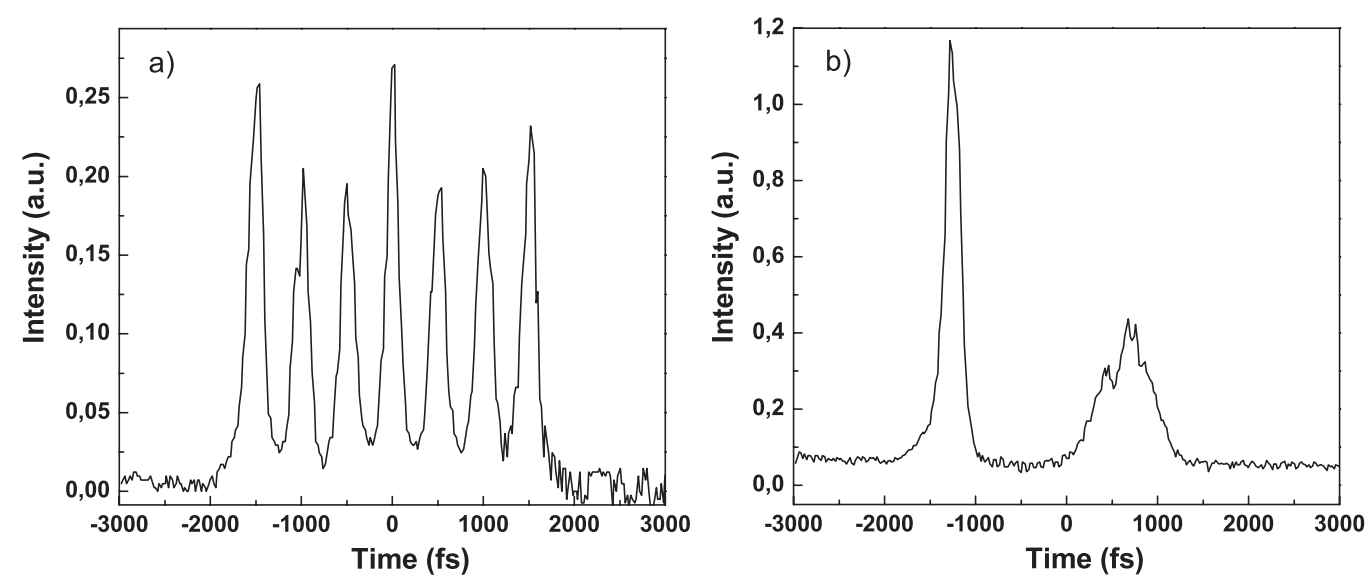

Figure 1. a) 7-pulse sequence at $268 \mathrm{~nm}$. b) Sequence of a Fourier-transform-limited pulse and a chirped pulse at $268 \mathrm{~nm}\left(\right.$ chirp $\left.=-11000 \mathrm{fs}^{2}\right)$. Both sequences have been characterized by DFG in a BBO crystal. 


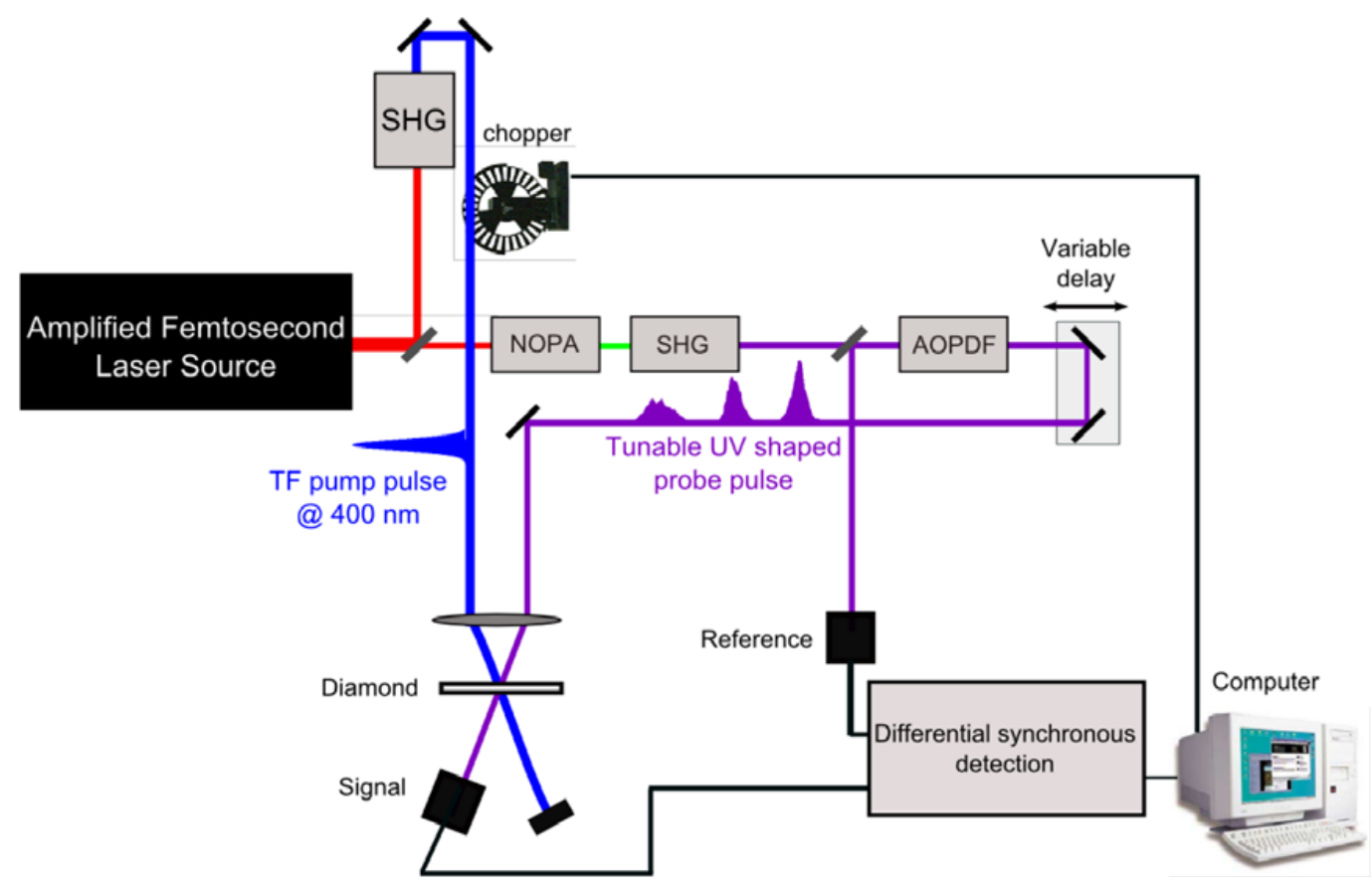

Figure 2. Experimental setup for characterization of shaped ultraviolet pulses by two photon absorption in diamond.

\section{SHAPING OF TUNABLE UV PULSES AND CHARACTERIZATION USING TWO-PHOTON ABSORPTION IN DIAMOND}

One main interest of this pulse shaper is the easy-way to use it on a broad spectral range. Unfortunately this is not the case for most of the usual characterization techniques based on non linear mixing which are limited by the phase-matching condition. To avoid this problem, it has been proposed to use degenerate four-wave mixing in fused silica [20] or two-photon absorption in diamond [21]. As far as we know, none of this techniques have been used to characterize complex shaped pulses. Combining the AOPDF technique and the TPA diamond characterization, we have obtained original results demonstrating the feasibility of this method.

In this set of experiments, either the second harmonic or the output of a frequency-doubled NOPA has been shaped by the AOPDF. The pulse characterization has been performed by detecting the two photon absorption transient transmission change using a conventional femtosecond pump-probe setup. To do so, a fraction of the CPA output has been frequency doubled to yield pump pulses at $408 \mathrm{~nm}$. Depending on the desired wavelength of the AOPDF input pulses, the remaining part is either directly frequency doubled $(408 \mathrm{~nm})$ or sent to a frequency-doubled Non Collinear Optical Parametric Amplifier [22] (tunable 250-350 nm pulses) before being injected into the AOPDF. The output of the AOPDF is the probe. Both pump and probe beams are then focused on $80 \mu \mathrm{m}$ thick monocrystalline diamond with a separate lens at an incidence angle of approximately $30^{\circ}$. The transient transmission change is detected by traditional differential detection technique. An autocorrelation measurement on the reference pump pulse gave a pulse duration of $60 \mathrm{fs}$. Then several complex shapes have been programmed at $408 \mathrm{~nm}$ and characterized by TPA in diamond (Fig. 2).

Figure 3(a) shows an example of a complex shape obtained at $400 \mathrm{~nm}$ with a 7 pulses sequence. The AOPDF allows both to compress the pulse from $150 \mathrm{fs}$ down to $70 \mathrm{fs}$ and to shape the pulse in phase and amplitude. The contrast is excellent. However a small increase of the background can be seen. 
a)

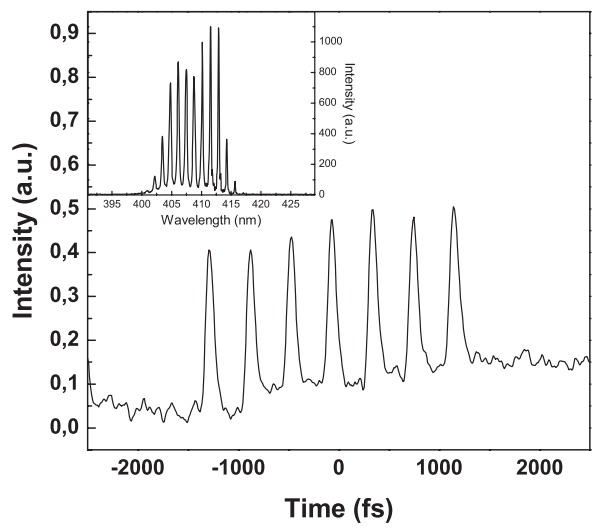

b)

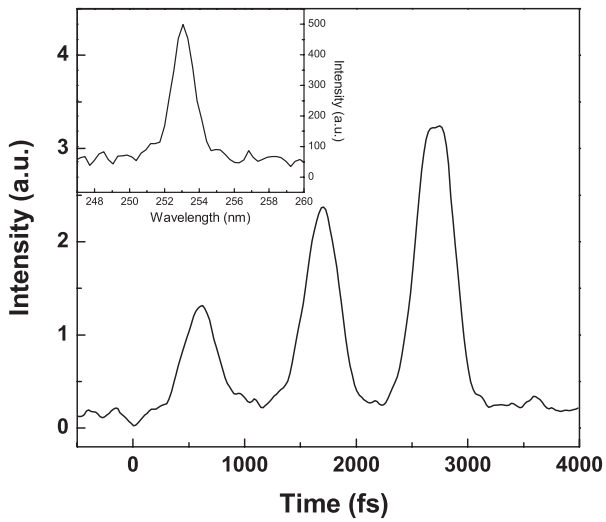

Figure 3. a) 7-pulse sequence at $408 \mathrm{~nm}$. b) 3-pulse sequence at $253 \mathrm{~nm}$. Both sequences have been characterized by two photon absorption in diamond. The corresponding spectra are given in insert. Due to insufficient resolution of our spectrometer and the delay between the pulses (1 ps), the fringes are not visible in the spectrum presented in $\mathrm{b}$.

This effect has been shown in [23] to be due to the ultrafast electronic response of diamond. Further work is under progress to fully characterize this effect. In fig. 3(b) very preliminary results at $253 \mathrm{~nm}$ are presented: Here pulses are not compressed but a 3-pulses sequence is programmed. The experiment was limited by the energy available at the output of the device but the improvement of the NOPA set-up as well as a careful adjustment of the diameter of the beam going through the AOPDF should allow to reduce this limitation. However we have demonstrated that it is possible to shape pulses at $253 \mathrm{~nm}$ and to measure them using the TPA in the diamond.

\section{CONCLUSION}

In this paper the results of pulse compression and shaping experiments are presented on a broad spectral range between $253 \mathrm{~nm}$ and $410 \mathrm{~nm}$. The performances in terms of temporal shaping window and tunability in the UV, with its high update rate make the AOPDF device with KDP material an ideal tool for a feedback loop in optimal control experiments. Moreover, the two-photon absorption diamond seems to be a very promising and easy-to-do method to characterize shaped pulses in this spectral range.

\section{Acknowledgments}

E. Baynard and S. Faure are acknowledged for their technical help. Charlotte Fabre is acknowledged for her help in the earlier stage of the work. This work has been supported by the Agence Nationale de la Recherche (Contract ANR - 06-BLAN-0004) and the Del Duca foundation.

\section{References}

[1] A. Assion, T. Baumert, M. Bergt, T. Brixner, B. Kiefer, V. Seyfried, M. Strehle, G. Gerber, Science 282, 919 (1998)

[2] H. Rabitz, R. De Vivie-Riedle, M. Motzkus, K.L. Kompa, Science 288, 824 (2000)

[3] R.J. Levis, G.M. Menkir, H. Rabitz, Science 292(5517), 709 (2001)

[4] J.L. Herek, W. Wohlleben, R.J. Cogdell, D. Zeidler, M. Motzkus, Nature 417(6888), 533 (2002)

[5] J. Degert, W. Wohlleben, B. Chatel, M. Motzkus, B. Girard, Phys. Rev. Lett. 89(20), 203003 (2002) 
[6] A. Monmayrant, B. Chatel, B. Girard, Phys. Rev. Lett. 96, 103002 (2006)

[7] N. Dudovich, D. Oron, Y. Silberberg, Phys. Rev. Lett. 88(12), 123004 (2002)

[8] A.M. Weiner, Rev. Sci. Instr. 71(5), 1929 (2000)

[9] G. Stobrawa, M. Hacker, T. Feurer, D. Zeidler, M. Motzkus, F. Reichel, Applied Physics B 72(5), 627 (2001)

[10] A. Monmayrant, B. Chatel, Rev. Sci. Instr. 75(8), 2668 (2004)

[11] D. Goswami, Phys. Rep. 374(6), 385 (2003)

[12] M. Dantus, V.V. Lozovoy, Chemical Reviews 104(4), 1813 (2004)

[13] M. Hacker, T. Feurer, R. Sauerbrey, T. Lucza, G. Szabo, Journal of the Optical Society of America B (Optical Physics) 18(6), 866 (2001)

[14] B. Pearson, T. Weinacht, optics express 17(7), 4385 (2007)

[15] F. Verluise, V. Laude, Z. Cheng, C. Spielmann, P. Tournois, Optics Letters 25(8), 575 (2000)

[16] M. Pittman, S. Ferré, J.P. Rousseau, L. Notebaert, J.P. Chambaret, G. Chériaux, Applied Physics B: Lasers and Optics 74(6), 529 (2002)

[17] A. Monmayrant, M. Joffre, T. Oksenhendler, R. Herzog, D. Kaplan, P. Tournois, Optics Letters 28(4), 278 (2003)

[18] A. Monmayrant, A. Arbouet, B. Girard, B. Chatel, B.J. Whitaker, A. Barman, D. Kaplan, Appl. Phys. B 81(2-3), 177 (2005), http://fr.arxiv.org/pdf/physics/0505152

[19] S. Coudreau, D. Kaplan, P. Tournois, Opt. Lett. 31(12), 1899 (2006)

[20] F.G. Omenetto, W.A. Schroeder, K. Boyer, J.W. Longworth, A. McPherson, C.K. Rhodes, Appl. Opt. 36(15), 3421 (1997)

[21] J. Dadap, G. Focht, D. Reitze, M. Downer, Opt. Lett. 16(7), 499 (1991)

[22] G. Cerullo, S. De Silvestri, Rev. Sci. Instr. 74(1), 1 (2003)

[23] T. Roth, R. Laenen, Opt. Commun. 189(4-6), 289 (2001) 